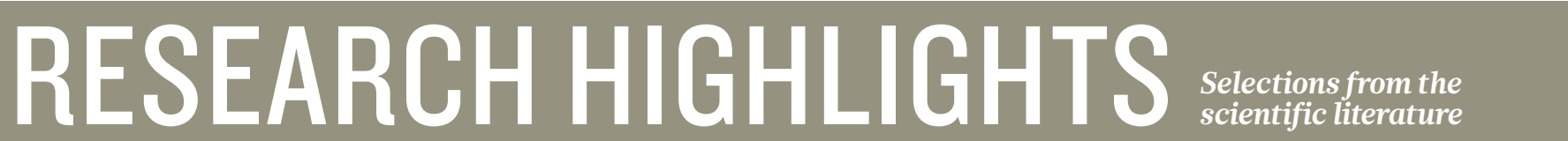

\section{PLANETARY SCIENCE}

\section{Signs of asteroid magnetic field}

The second most massive asteroid in the Solar System, Vesta, may once have had a rotating liquid metallic core that generated a magnetic field at the asteroid's surface.

Space scientists had previously established that the 525-kilometre-wide rock had differentiated - forming a dense core and a lighter mantle and crust. Roger Fu at the Massachusetts Institute of Technology in Cambridge and his colleagues studied the meteorite Allan Hills A81001, which probably originated on Vesta, and detected signatures of a magnetic field that formed 3.69 billion years ago. The team surmises that Vesta's rotating core produced a dynamo powerful enough to generate a magnetic field with a strength of 10 to 100 microteslas.

The discovery suggests that dynamos might have formed in other small, differentiated bodies in the early Solar System.

Science 338, 238-241 (2012)

\section{CANCER}

\section{Fat helps tumours to grow}

Researchers have found one possible explanation for the link between obesity and poor cancer prognosis: cells from fat tissue are recruited to the blood vessels that feed tumours, actively supporting tumour growth.

Mikhail Kolonin and his colleagues at the University of Texas Health Science Center at Houston grafted tumour cells into obese and lean mice that were fed the same diet. Tumours grew faster in the obese mice than in the

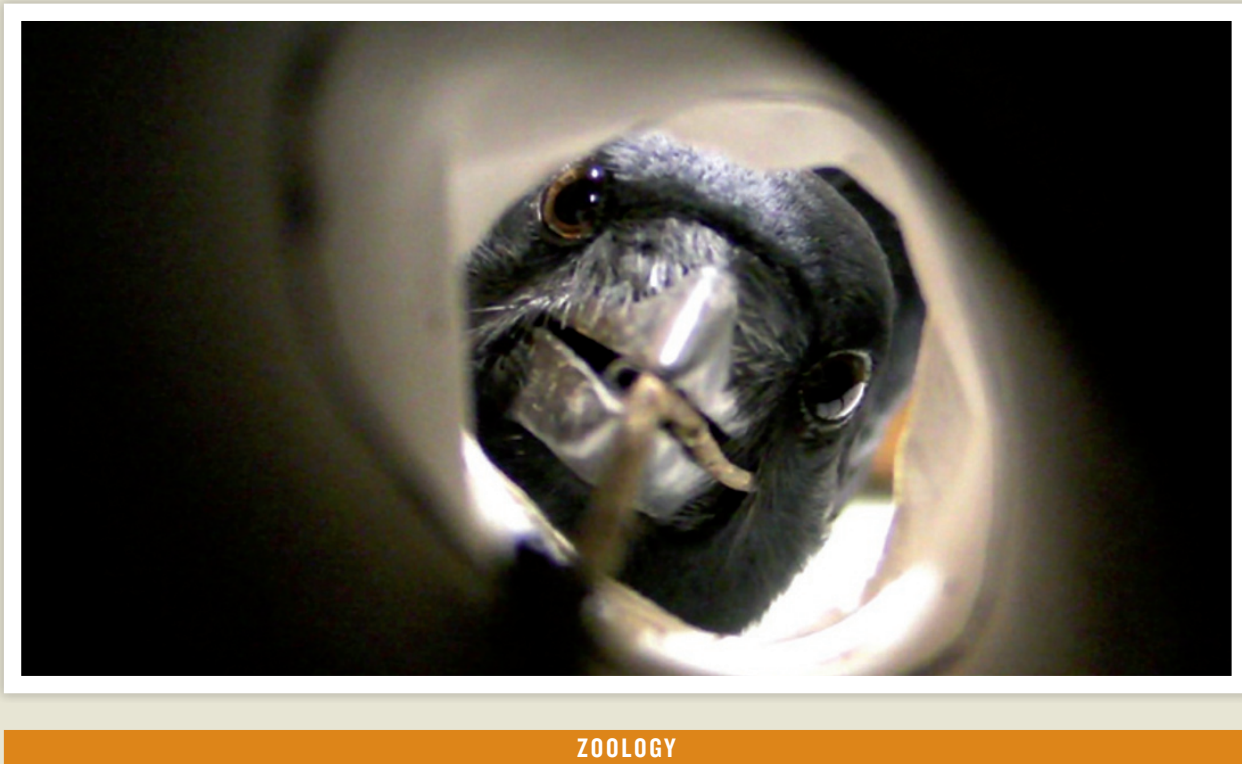

\section{Tool use takes more than brains}

Skilled tool use is considered a sign of intelligence, but a study of crafty crows shows that it takes the right physical attributes too.

New Caledonian crows (Corvus moneduloides; pictured) extract prey from nooks and crannies with tools that they craft from sticks and other items. Jolyon Troscianko at the University of Birmingham and Christian Rutz now at the University of St Andrews, both in the UK, and their colleagues studied the birds' visual fields and bill shapes. Compared with related species that do not use tools, New Caledonian crows have a larger field of binocular overlap. Using a video camera in the base of a baited tube, the team found that $\mathrm{New}$ Caledonian crows bring both eyes forward during tool use, which allows them greater visual control. Moreover, the straighter bill of C. moneduloides compared to related species gives the bird better control over a tool within its field of binocular vision.

Like the flexible wrists and opposable thumbs of humans, these traits provide a rare example of physical adaptations for tool use.

Nature Commun. http://dx.doi.org/10.1038/ ncomms2111 (2012) lean ones. The researchers found that fat-progenitor cells were mobilized into the bloodstream in the obese mice and were more abundant in the tumours of these animals than in those of their lean counterparts. In the tumours, the progenitors developed into fat cells and cells that support blood vessels. Tumours with a greater contribution from fat cells had a more extensive and functional vasculature, and grew faster.

Cancer Res. 72, 5198-5208 (2012)

\section{CELL THERAPY \\ Stem cells wrap neurons}

Human neural stem cells transplanted into the brain can improve the functioning of nerve cells by supplying myelin - which sheathes neurons and aids electrical signalling according to two independent groups.

David Rowitch at the University of California, San Francisco, and his colleagues transplanted the stem cells into four young boys with a genetic disease in which support cells known as oligodendrocytes fail to wrap neurons in myelin. A year after injection, magnetic resonance imaging (MRI) indicated that the transplanted cells had engrafted and had successfully myelinated brain cells.

Researchers led by Stephen Back at Oregon Health and Science University in Portland and Nobuko Uchida at StemCells Inc. in Newark, California, showed that the same type of stem cell matured into oligodendrocytes when transplanted into the brains 